\title{
SETS OF UNIQUENESS, WEAKLY SUFFICIENT SETS AND SAMPLING SETS FOR $A^{-\infty}(\mathbb{B})$
}

\author{
Le Hai KHOI
}

\begin{abstract}
We study a relationship between sets of uniqueness, weakly sufficient sets and sampling sets in the space $A^{-\infty}(\mathbb{B})$ of holomorphic functions with polynomial growth on the unit ball of $\mathbb{C}^{n}(n \geq 1)$.
\end{abstract}

\section{Introduction}

Let $\mathcal{O}(\mathbb{B}), \mathbb{B}$ being the open unit ball in $\mathbb{C}^{n}$ for some integer $n \geq 1$, denote the set of holomorphic functions defined on $\mathbb{B}$, with the usual compact-open topology. The space $A^{-\infty}=A^{-\infty}(\mathbb{B})$ of holomorphic functions on $\mathbb{B}$ with the polynomial-growth condition, is defined as follows

$$
A^{-\infty}(\mathbb{B}):=\left\{f \in \mathcal{O}(\mathbb{B}): \exists p, C>0, \sup _{z \in \mathbb{B}}(1-|z|)^{p}|f(z)| \leq C\right\} .
$$

As is well known, $A^{-\infty}$ is the smallest algebra of holomorphic functions that contains the class $H^{\infty}$ of bounded holomorphic functions and is closed under differentiation.

It should be noted that the space $A^{-\infty}(\mathbb{B})$ has been studied intensively by many mathematicians (C. A. Horowitz, B. Korenblum, B. Pinchuk; X. Massaneda; J. Ortega; P. Thomas, J. Bonet, P. Domański, etc - see references). Most work concentrated on interpolating sequences, and for the onedimensional case $A^{-\infty}(\mathbb{D})$, also on sampling sets.

The goal of this article is to study relationship between various sets, such as sets of uniqueness, weakly sufficient sets and sampling sets, in the space $A^{-\infty}$.

Received March 5, 2009; Revised July 12, 2010.

2000 Mathematics Subject Classification. 32A38, 46A13.

Key words and phrases. sampling set, weakly sufficient set, set of uniqueness, function algebra, polynomial growth.

The main idea of this work was formed during the author's visit at POSTECH. He would like to express his thanks to POSTECH for its hospitality. 


\section{Basic definitions}

\subsection{Weakly sufficient sets}

Let $f \in \mathcal{O}(\mathbb{B})$. Let $p$ be a positive real number. Define

$$
\|f\|_{p}:=\sup _{z \in \mathbb{B}}(1-|z|)^{p}|f(z)|,
$$

and

$$
A^{-p}=A^{-p}(\mathbb{B}):=\left\{f \in \mathcal{O}(\mathbb{B}):\|f\|_{p}<+\infty\right\}
$$

Notice that

$$
A^{-\infty}=\bigcup_{p>0} A^{-p}
$$

We can endow $A^{-\infty}$ with a topological structure, namely the internal inductive limit of spaces $\left\{\left(A^{-p},\|\|_{p}\right): p>0\right\}$ and denote it by

$$
\left(A^{-\infty}, \tau\right)=\lim _{p \rightarrow 0}\left(A^{-p},\|\|_{p}\right) \text {, or briefly, }\left(A^{-\infty}, \tau\right)=\lim \text { ind } A^{-p} \text {. }
$$

Now let $S$ be a subset of $\mathbb{B}$. Define

$$
\|f\|_{p, S}:=\sup _{z \in S}(1-|z|)^{p}|f(z)|
$$

and

$$
A^{-p, S}=A^{-p, S}(\mathbb{B}):=\left\{f \in A^{-\infty}:\|f\|_{p, S}<+\infty\right\} .
$$

Notice that \|\|$_{p, S}$ is in general a semi-norm, and that the inclusion relations $A^{-p} \subset A^{-p, S} \subset A^{-\infty}$ hold. Hence, it follows immediately that

$$
A^{-\infty}=\bigcup_{p>0} A^{-p} \subset \bigcup_{p>0} A^{-p, S} \subset A^{-\infty}
$$

and consequently that

$$
A^{-\infty}=\bigcup_{p>0} A^{-p}=\bigcup_{p>0} A^{-p, S} .
$$

Moreover, it is obvious that $A^{-p} \hookrightarrow A^{-p, S}$ (here and in a sequel the symbol $\hookrightarrow$ denotes a continuous imbedding). Therefore, for an arbitrarily given subset $S \subset \mathbb{B}$, one can construct on $A^{-\infty}$ another (weaker, and in fact strictly weaker in many cases) internal inductive limit topology from the sequence $\left(A^{-p, S},\|\|_{p, S}\right)$ of vector spaces equipped with the semi-norms. Namely,

$$
\left(A^{-\infty}, \tau_{S}\right)=\lim _{\vec{p}>0}\left(A^{-p, S},\|\|_{p, S}\right) \text {, or briefly, }\left(A^{-\infty}, \tau_{S}\right)=\lim \text { ind } A^{-p, S} .
$$

Definition 2.1. A subset $S \subset \mathbb{B}$ is said to be a weakly sufficient set for the space $A^{-\infty}(\mathbb{B})$ if two topologies $\tau$ and $\tau_{S}$ are equivalent. 
Therefore it is quite surprising that there is a discrete subset $S$ of $\mathbb{B}$ which is weakly sufficient, for instance.

In our previous paper [3] we gave the presentation of an explicit method, even an "algorithm", for the construction of countable, weakly sufficient sets for $A^{-\infty}$ for every $n \geq 1$. In fact, it was considered a more general case than the unit ball, namely, for a bounded domain with $C^{1}$ smooth boundary. This is the starting point of our investigation on various sets in the space $A^{-\infty}$.

\subsection{Sets of uniqueness}

Furthermore, in function spaces there is a quite well-known notion of a set of uniqueness. For the space $A^{-\infty}$ it can be defined as follows.

Definition 2.2. A subset $S \subset \mathbb{B}$ is called a set of uniqueness for $A^{-\infty}(\mathbb{B})$ if $f \in A^{-\infty}$ and $f(z)=0$ for all $z \in S$ imply that $f=0$.

Thus sets of uniqueness are precisely those sets which are not subsets of zero-sets for $A^{-\infty}$. Below we can see that the uniqueness property is necessary for a set to be weakly sufficient in $A^{-\infty}$.

\subsection{Sampling sets}

We notice that in the one-dimensional case the so-called sampling sets in the space $A^{-\infty}(\mathbb{D}), \mathbb{D}$ being the open unit disc in $\mathbb{C}$, were introduced and studied intensively by many people (see, e.g., [4], and references therein, also $[6],[2], \ldots)$. It seems that this notion is the same as for effective sets introduced by Iyer in [5].

Inspiring by those works, for $f \in A^{-\infty}$, we consider

$$
T_{f}=\limsup _{|z| \rightarrow 1} \frac{\log |f(z)|}{|\log (1-|z|)|} .
$$

If $S$ is a subset of $\mathbb{B}$, we define

$$
T_{f, S}=\limsup _{\substack{|z| \rightarrow 1 \\ z \in S}} \frac{\log |f(z)|}{|\log (1-|z|)|} .
$$

It is obvious from the definition that $T_{f, S} \leq T_{f}$ for any $S \subset \mathbb{B}$ and $f \in A^{-\infty}(\mathbb{B})$. Our interest is on the question: for which subsets $S$ of $\mathbb{B}$ the reverse inequality can hold? In fact the following notion of sampling set has been studied for some time.

Definition 2.3. A subset $S$ of $\mathbb{B}$ is called a sampling set for $A^{-\infty}(\mathbb{B})$, if the equality $T_{f}=T_{f, S}$ holds for every $f \in A^{-\infty}(\mathbb{B})$.

In $[6]$, the following implications in $A^{-\infty}(\mathbb{D})$ were proven: the sampling property implies the weak sufficiency property, and the weak sufficiency property in turn implies uniqueness property. Examples showing that the converse implications are not true were also provided. 
It is of interest to study the relationship between sets of uniqueness, weakly sufficient sets, and sampling sets in the space $A^{-\infty}$ for higher dimension.

From now on, instead of a family $\{p>0\}$, we restrict ourselves to take $p \in \mathbb{N}$, i.e., the space $A^{-\infty}(\mathbb{B})$ is the countable union of a sequence of Banach spaces:

$$
A^{-\infty}(\mathbb{B})=\bigcup_{p=1}^{\infty} A^{-p}(\mathbb{B}) .
$$

\section{Weakly sufficient sets and sets of uniqueness}

First we make some characterizations of the weakly sufficient sets and sets of uniqueness in the space $A^{-\infty}$ that are needed in the sequel.

Proposition 3.1 (see [3]). For the space $A^{-\infty}(\mathbb{B})$ the following statements are equivalent:

(a) $S$ is a weakly sufficient set, i.e., $\tau_{S}=\tau$.

(b) For every $p>0$, there exists $m=m(p)>0$ such that

$$
\left(A^{-p, S},\|\|_{p, S}\right) \hookrightarrow\left(A^{-m},\|\|_{m}\right),
$$

that is,

$\forall p>0 \exists m=m(p), C=C(p)>0:\|f\|_{m} \leq C\|f\|_{p, S}, \forall f \in A^{-p, S}$.

(c) For every $p>0$ there exist $m=m(p)>0$ and $C=C(p)>0$ such that

$$
\|f\|_{m} \leq C\|f\|_{p, S}, \forall f \in A^{-\infty} .
$$

Also we note the following result.

Remark 3.2. For the space $A^{-\infty}(\mathbb{B})$ the following statements are equivalent:

(a) $S$ is a set of uniqueness.

(b) $A^{-p, S}$ is a normed space (for all $p>0$ ).

Now we are able to prove the following relationship.

Proposition 3.3. Any weakly sufficient set for $A^{-\infty}(\mathbb{B})$ is a set of uniqueness for this space.

Proof. Let $S \subset \mathbb{B}$ be a weakly sufficient set for $A^{-\infty}$, i.e., $\left(A^{-\infty}, \tau_{S}\right)=$ $\left(A^{-\infty}, \tau\right)$. If $f \in A^{-\infty}$ with $f(z)=0, \forall z \in S$, then for any $p>0$ we have

$$
\sup _{z \in S}(1-|z|)^{p}|f(z)|=0 \text {, }
$$

which means that $f \in A^{-p, S}$ and $\|f\|_{p, S}=0, \forall p>0$. If $A=(\alpha)$ is a family of seminorms defining a topology in $\left(A^{-\infty}, \tau_{S}\right)$, then since $A^{-p, S} \hookrightarrow\left(A^{-\infty}, \tau_{S}\right)$, from $\|f\|_{p, S}=0, \forall p>0$ it follows that $\alpha(f)=0, \forall \alpha \in A$. But two topologies $\tau_{S}$ and $\tau$ are equivalent, the last equality shows that $\beta(f)=0, \forall \beta \in B$, where $B=(\beta)$ is a family of seminorms defining a topology in $\left(A^{-\infty}, \tau\right)$, and therefore, $f=0$, by the fact that the space $\left(A^{-\infty}, \tau\right)$ is separated. 
Here we note that Proposition 3.3 can also be proved by contradiction: if two distinct functions coincide on $S$, it is easy to build a non convergent sequence in $A^{-\infty}$ that always converges in the " $S$-topology".

In general, a set of uniqueness for $A^{-\infty}$ is not necessarily weakly sufficient for this space. However, it turns out that under some quite surprising conditions the converse is true. Below we present the converse result, and moreover, in two parts, the necessity, whose proof is quite simple, and the sufficiency which requires a rather "delicate" proof.

\subsection{The necessary conditions}

Combining Propositions 3.1 and 3.3, we see that if $S$ is a weakly sufficient set for $A^{-\infty}(\mathbb{B})$, then

(a) $S$ is a set of uniqueness for this space.

(b) The following set-inclusion holds

$$
\forall p>0 \exists m=m(p)>0: A^{-p, S} \subset A^{-m} .
$$

Notice that in the one-dimensional case $n=1$ the set-inclusion condition (3) implies that $S$ is a set of uniqueness for the space $A^{-\infty}(\mathbb{D})$ (see [6]). It was also proved that (3) implies the continuous imbedding, which means that this condition is equivalent to the weak sufficiency of a set $S$.

\subsection{Sufficient conditions}

We now consider a question when a set of uniqueness will be weakly sufficient. It turns out that the converse of the conditions above holds.

Theorem 3.4. Suppose that

(a) $S$ is a set of uniqueness for $A^{-\infty}(\mathbb{B})$, and

(b) the condition (3) holds, i.e., $\forall p \exists m=m(p): A^{-p, S} \subset A^{-m}$.

Then $S$ is a weakly sufficient set for $A^{-\infty}(\mathbb{B})$.

Proof. Let $K_{s}=\left\{z \in \mathbb{B}:|z| \leq r_{s}\right\}$ where $r_{s}$ satisfies the conditions:

(i) $0<r_{s}<r_{t}<1$ for $0<s<t$, and

(ii) $\lim _{s \rightarrow \infty} r_{s}=1$.

It is obvious that the family $\left\{K_{s}: s>0\right\}$ consists of compact subsets of the unit ball $\mathbb{B}$ such that $K_{s} \subset \subset K_{t}$ for $0<s<t$ and $\bigcup_{s>0} K_{s}=\mathbb{B}$.

By the second assumption of the theorem, take any $p \geq 1$, then $\exists m=m(p)$ such that $A^{-p, S} \subset A^{-m}$. As $A^{-m} \subset A^{-(m+1)}, \forall m \geq 1$, without lost of generality, we can consider that $m \geq p$. It suffices, by Proposition 3.1, to prove that $A^{-p, S}$ is imbedded continuously into some space $A^{-q}$ with $q>m \geq p$.

Let $E_{p, S}$ be the unit ball in $A^{-p, S}$. Let $q>m$. Then $q \geq m+1 \geq p+1$. Denote by $E_{q}$ the unit ball in $A^{-q}$, then as

$$
\sup _{f \in E_{p, S}}\|f\|_{q} \leq \sup _{f \in E_{p, S} \backslash E_{q}}\|f\|_{q}+\sup _{f \in E_{q}}\|f\|_{q},
$$


we need only to prove that $E=E_{p, S} \backslash E_{q}$ is bounded in the space $A^{-q}$.

At this point we pause and prove the following result for the norm $\|f\|_{q}$.

Lemma 3.5. The $\|f\|_{q}$ is attained on some compact subset $K_{s} \subset \mathbb{B}$ for all $f \in E$.

Proof. Expecting a contradiction we suppose that the statement of the lemma is not true. This means that

$$
\forall K_{s} \exists f \in E: \sup _{K_{s}}(1-|z|)^{q}|f(z)|<\|f\|_{q},
$$

from which it follows that

$$
\sup _{\mathbb{B} \backslash K_{s}}(1-|z|)^{q}|f(z)| \geq\|f\|_{q} .
$$

Our aim is to construct, employing the methods of [1] and [6], a function $h$ such that $h \in A^{-q}$, but $h \notin A^{-(q-1)}$. Moreover, this function is constructed in the form of a series $h(z)=\sum_{k=1}^{\infty} c_{k} h_{k}(z)$, where $h_{k} \in \mathcal{E}:=\left\{\frac{f}{\|f\|_{q}} ; f \in E\right\}$ will be defined inductively as follows.

Take an arbitrary $h_{1} \in \mathcal{E}$. If we have $h_{1}, \ldots, h_{s-1} \in \mathcal{E}(s \geq 2)$, then $h_{s}$ is determined in the following way.

- First, choose $K_{s} \subset \mathbb{B}$ large enough so that

$$
1-|z| \leq \frac{1}{3 \cdot 4^{s+1} \sum_{k=1}^{s-1}\left\|h_{k}\right\|_{q-1}}, \forall z \in \mathbb{B} \backslash K_{s} .
$$

- Next, for such a $K_{s}$, there is an $f_{s} \in E$ such that (4) is satisfied, and we define $h_{s}(z)=\frac{f_{s}(z)}{\left\|f_{s}\right\|_{q}} \in \mathcal{E}$.

Here we note that, by (4) there exists $z_{s} \in \mathbb{B} \backslash K_{s}$ such that

$$
\left(1-\left|z_{s}\right|\right)^{q}\left|f_{s}\left(z_{s}\right)\right| \geq \frac{\left\|f_{s}\right\|_{q}}{2} .
$$

Thus a sequence $\left(h_{k}\right) \subset \mathcal{E}$ is defined. Taking $c_{k}=4^{-k}(k=1,2, \ldots)$, for $h(z)=\sum_{k=1}^{\infty} 4^{-k} h_{k}(z)$ we have

$$
\|h\|_{q} \leq \sum_{k=1}^{\infty} \frac{1}{4^{k}}\left\|h_{k}\right\|_{q}=\sum_{k=1}^{\infty} \frac{1}{4^{k}}<\infty,
$$

which means that $h \in A^{-q}$, and therefore, $h \in A^{-\infty}$.

On the other hand,

$$
\begin{aligned}
\left|h\left(z_{s}\right)\right| & =\left|\sum_{k=1}^{\infty} \frac{1}{4^{k}} h_{k}\left(z_{s}\right)\right| \\
& \geq\left|\frac{1}{4^{s}} h_{s}\left(z_{s}\right)\right|-\left|\sum_{k \neq s}^{\infty} \frac{1}{4^{k}} h_{k}\left(z_{s}\right)\right|
\end{aligned}
$$




$$
\begin{aligned}
& \geq \frac{1}{4^{s}}\left|h_{s}\left(z_{s}\right)\right|-\sum_{k \neq s}^{\infty} \frac{1}{4^{k}}\left|h_{k}\left(z_{s}\right)\right| \\
& =\frac{1}{4^{s}}\left|h_{s}\left(z_{s}\right)\right|-\sum_{k=1}^{s-1} \frac{1}{4^{k}}\left|h_{k}\left(z_{s}\right)\right|-\sum_{k=s+1}^{\infty} \frac{1}{4^{k}}\left|h_{k}\left(z_{s}\right)\right| .
\end{aligned}
$$

Furthermore, we note the following estimations:

- By (6)

$$
\left|h_{s}\left(z_{s}\right)\right|=\frac{\left|f_{s}\left(z_{s}\right)\right|}{\left\|f_{s}\right\|_{q}} \geq \frac{1}{2}\left(1-\left|z_{s}\right|\right)^{-q} .
$$

- Also $\forall k=1, \ldots, s-1$

(9)

$$
\begin{aligned}
\left|h_{k}\left(z_{s}\right)\right| & =\left\{\left(1-\left|z_{s}\right|\right)^{q-1}\left|h_{k}\left(z_{s}\right)\right|\right\}\left(1-\left|z_{s}\right|\right)^{-(q-1)} \\
& \leq \sup _{z \in \mathbb{B}}\left\{(1-|z|)^{q-1}\left|h_{k}(z)\right|\right\}\left(1-\left|z_{s}\right|\right)^{-(q-1)} \\
& =\left\|h_{k}\right\|_{q-1}\left(1-\left|z_{s}\right|\right)^{-(q-1)}
\end{aligned}
$$

- Similarly, $\forall k \geq s+1$

$$
\begin{aligned}
\left|h_{k}\left(z_{s}\right)\right| & =\left\{\left(1-\left|z_{s}\right|\right)^{q}\left|h_{k}\left(z_{s}\right)\right|\right\}\left(1-\left|z_{s}\right|\right)^{-q} \\
& \leq \sup _{z \in \mathbb{B}}\left\{(1-|z|)^{q}\left|h_{k}(z)\right|\right\}\left(1-\left|z_{s}\right|\right)^{-q} \\
& =\left\|h_{k}\right\|_{q}\left(1-\left|z_{s}\right|\right)^{-q}=\left(1-\left|z_{s}\right|\right)^{-q}
\end{aligned}
$$

as $\left\|h_{k}\right\|_{q}=1, \forall k \geq 1$.

Combining (8)-(10) we can continue (7) as follows

$$
\begin{aligned}
& \left|h\left(z_{s}\right)\right| \\
\geq & \frac{1}{4^{s}} \cdot \frac{1}{2}\left(1-\left|z_{s}\right|\right)^{-q}-\sum_{k=1}^{s-1} \frac{1}{4^{k}}\left\|h_{k}\right\|_{q-1}\left(1-\left|z_{s}\right|\right)^{-(q-1)}-\sum_{k=s+1}^{\infty} \frac{1}{4^{k}}\left(1-\left|z_{s}\right|\right)^{-q} \\
= & \left(\frac{1}{2 \cdot 4^{s}}-\sum_{k=s+1}^{\infty} \frac{1}{4^{k}}\right) \cdot\left(1-\left|z_{s}\right|\right)^{-q}-\left(1-\left|z_{s}\right|\right)^{-(q-1)} \sum_{k=1}^{s-1} \frac{1}{4^{k}}\left\|h_{k}\right\|_{q-1} \\
= & \frac{1}{6 \cdot 4^{s}}\left(1-\left|z_{s}\right|\right)^{-q}-\left(1-\left|z_{s}\right|\right)^{-(q-1)} \sum_{k=1}^{s-1} \frac{1}{4^{k}}\left\|h_{k}\right\|_{q-1} \\
\geq & \frac{1}{6 \cdot 4^{s}}\left(1-\left|z_{s}\right|\right)^{-(q-1)}\left(3 \cdot 4^{s+1} \sum_{k=1}^{s-1}\left\|h_{k}\right\|_{q-1}\right)-\left(1-\left|z_{s}\right|\right)^{-(q-1)} \sum_{k=1}^{s-1}\left\|h_{k}\right\|_{q-1},
\end{aligned}
$$

by (5), that is,

$$
\left|h\left(z_{s}\right)\right| \geq\left(1-\left|z_{s}\right|\right)^{-(q-1)} \sum_{k=1}^{s-1}\left\|h_{k}\right\|_{q-1} .
$$


As $\left\|h_{k}\right\|_{q-1} \geq\left\|h_{k}\right\|_{q}=1, \forall k \geq 1$, from (11) it follows that

$$
\begin{aligned}
\|h\|_{q-1} & =\sup _{z \in \mathbb{B}}(1-|z|)^{q-1}|h(z)| \\
& \geq\left(1-\left|z_{s}\right|\right)^{q-1}\left|h\left(z_{s}\right)\right| \\
& \geq \sum_{k=1}^{s-1}\left\|h_{k}\right\|_{q-1} \\
& \geq s-1, \forall s \geq 2,
\end{aligned}
$$

which implies that $\|h\|_{q-1}=\infty$. We have thus obtained that $h \in A^{-\infty}$, but $h \notin A^{-(q-1)}$.

Now turn back to the definition of the set $E=E_{p, S} \backslash E_{q}$. We see that if $f \in E$, then $\|f\|_{p, S} \leq 1$ and $\|f\|_{q}>1$. Hence, for any $g \in \mathcal{E}=\left\{\frac{f}{\|f\|_{q}} ; f \in E\right\}$ we have $\|g\|_{p, S}<1$. From this fact it follows that for the function $h$ constructed above, we have the following estimation

$$
\|h\|_{p, S} \leq \sum_{k=1}^{\infty} \frac{1}{4^{k}}\left\|h_{k}\right\|_{p, S} \leq \sum_{k=1}^{\infty} \frac{1}{4^{k}}<\infty,
$$

which means that $h \in A^{-p, S}$.

Thus $h \in A^{-p, S}$, and $h \notin A^{-(q-1)}$, while by the assumption of the theorem $A^{-p, S} \subset A^{-m} \subset A^{-(q-1)}$ (as $\left.p \leq m \leq q-1\right)$ : a contradiction. The lemma is proved.

From Lemma 3.5 using the regularity property of the space $A^{-\infty}$ (see [3], also [7], [10]) we can derive boundedness of $E_{p, S}$. Indeed, we have

$$
\begin{aligned}
\forall f \in E:\|f\|_{m} & =\sup _{z \in \mathbb{B}}(1-|z|)^{m}|f(z)|=\sup _{z \in K_{s}}(1-|z|)^{m}|f(z)| \\
& =\sup _{z \in K_{s}}\left\{(1-|z|)^{m+1}|f(z)| \cdot \frac{1}{(1-|z|)}\right\} \\
& \leq \frac{1}{\left(1-r_{s}\right)} \cdot \sup _{z \in K_{s}}(1-|z|)^{m+1}|f(z)| \leq \frac{1}{\left(1-r_{s}\right)} \cdot\|f\|_{m+1} .
\end{aligned}
$$

The last inequality means that a set

$$
\left\{\frac{f}{\|f\|_{m+1}}\right\}_{f \in E}
$$

is bounded in $A^{-m}$, and therefore, it is a relatively compact subset in $A^{-(m+1)}$, by the complete continuity of the imbedding of $A^{-m}$ into $A^{-(m+1)}$.

Furthermore, we have

$$
\sup _{f \in E}\|f\|_{m+1, S} \leq \sup _{f \in E}\|f\|_{p, S} \leq 1 .
$$


Taking into account that $S$ is a set of uniqueness for the space $A^{-(m+1)}$, from the last two facts it follows that $E$ is bounded in $A^{-(m+1)}$, and therefore, is bounded in $A^{-m}$, by (12).

Indeed, denote $C=\sup _{f \in E}\|f\|_{m+1}$, which implies that there is a sequence $\left(f_{k}\right) \subset E$ such that

$$
\lim _{k \rightarrow \infty}\left\|f_{k}\right\|_{m+1}=C .
$$

Also, by (13), for a sequence $\left(f_{k} /\left\|f_{k}\right\|_{m+1}\right) \subset E \subset A^{-(m+1)}$ there is a subsequence $\left(g_{\ell}=f_{k_{\ell}} /\left\|f_{k_{\ell}}\right\|_{m+1}\right)$ such that it converges to some element, denoted by $\widehat{f}$ of $A^{-(m+1)}$.

As $\left\|g_{\ell}\right\|_{m+1}=1, \forall \ell \geq 1$, we get that $\|\widehat{f}\|_{m+1}=1$, too. This means that $\widehat{f}$ is a nonzero element in $A^{-(m+1)}$. Then, by the assumption of the theorem, $S$ is a set of uniqueness of the space $A^{-(m+1)}$ it holds $\|\widehat{f}\|_{m+1, S}=C_{1}>0$.

Taking into account that

$$
\left\|g_{\ell}-\widehat{f}\right\|_{m+1, S} \leq\left\|g_{\ell}-\widehat{f}\right\|_{m+1} \text { and } \lim _{\ell \rightarrow \infty}\left\|g_{\ell}-\widehat{f}\right\|_{m+1}=0,
$$

it follows that $\lim _{\ell \rightarrow \infty}\left\|g_{\ell}-\widehat{f}\right\|_{m+1, S}=0$, too. Hence, for all $\ell$ big enough we have

$$
\left\|g_{\ell}\right\|_{m+1, S} \geq \frac{\|\widehat{f}\|_{m+1, S}}{2}=\frac{C_{1}}{2}
$$

On the other hand,

$$
\left\|g_{\ell}\right\|_{m+1, S}=\left\|\frac{f_{k_{\ell}}}{\left\|f_{k_{\ell}}\right\|_{m+1}}\right\|_{m+1, S}=\frac{\left\|f_{k_{\ell}}\right\|_{m+1, S}}{\left\|f_{k_{\ell}}\right\|_{m+1}} \leq \frac{1}{\left\|f_{k_{\ell}}\right\|_{m+1}},
$$

by (14).

From (16)-(17) it follows that

$$
\left\|f_{k_{\ell}}\right\|_{m+1} \leq \frac{1}{\left\|g_{\ell}\right\|_{m+1, S}} \leq \frac{2}{C_{1}}
$$

Hence, by (15)

$$
\sup _{f \in E}\|f\|_{m+1}=C=\lim _{\ell \rightarrow \infty}\left\|f_{k_{\ell}}\right\|_{m+1} \leq \frac{2}{C_{1}}<\infty,
$$

which means that $E$ is bounded in $A^{-(m+1)}$.

Thus $A^{-p, S} \hookrightarrow A^{-(m+2)}$, and $S$ is a weakly sufficient set for $A^{-\infty}$, by Proposition 3.1.

Thus we have proved the following result.

Theorem 3.6. A set $S \subset \mathbb{B}$ is weakly sufficient for $A^{-\infty}(\mathbb{B})$ if and only if $S$ is a set of uniqueness for $A^{-\infty}(\mathbb{B})$, and $\forall p \exists m=m(p): A^{-p, S} \subset A^{-m}$.

In other words, Theorem 3.6 states that for a set of uniqueness in the space $A^{-\infty}$, a set-inclusion implies a continuous imbedding. 


\section{Sampling sets and weakly sufficient sets}

In this section we study some questions on sampling sets and their relationship to weakly sufficient sets.

\subsection{A toy problem for the non-discrete case}

First we prove that a union of concentric to $\mathbb{B}$ spheres forms a (non-discrete) sampling set for the space $A^{-\infty}$. The "distances" between spheres are supposed to satisfy some conditions.

Let $0<\left(t_{k}\right) \uparrow 1$ and

$$
\lim _{k \rightarrow \infty} \frac{\left|\log \left(1-t_{k+1}\right)\right|}{\left|\log \left(1-t_{k}\right)\right|}=1 .
$$

Such sequences are abundant. For example, $t_{k}=1-\frac{1}{(k+1)^{\alpha}}, \alpha>0$, satisfies that condition; also $t_{k}=1-e^{-e^{\sqrt{k}}}$ works, ....

Denote by

$$
\mathcal{S}_{k}=\left\{z \in \mathbb{B}:|z|=t_{k}\right\}
$$

and let

$$
\mathcal{S}=\bigcup_{k=1}^{\infty} \mathcal{S}_{k}
$$

Notice that for the case $n=1$ in [4] it was proved that the condition (18) is necessary and sufficient for the set $\mathcal{S}$ to be sampling for $A^{-\infty}(\mathbb{D})$.

Following that method we can prove that in higher dimension the same union works well, too.

Proposition 4.1. $S$ is a sampling set for $A^{-\infty}$.

Proof. Let $f \in A^{-\infty}(\mathbb{B})$, and let $\varepsilon>0$ be given. Then from (18) as well as the definition of $T_{f, S}$ it follows that $\exists N$ such that $\forall k>N$ :

$$
\frac{\left|\log \left(1-t_{k+1}\right)\right|}{\left|\log \left(1-t_{k}\right)\right|}<1+\varepsilon
$$

and

$$
\sup _{|z|=t_{k}} \frac{\log |f(z)|}{\left|\log \left(1-t_{k}\right)\right|}<T_{f, S}+\varepsilon .
$$

Now for $k>N$ and $t_{k} \leq|z|<t_{k+1}$ we have

$$
\begin{aligned}
\frac{\log |f(z)|}{|\log (1-|z|)|} & \leq \sup _{|w|=t_{k+1}} \frac{\log |f(w)|}{\left|\log \left(1-t_{k}\right)\right|} \\
& =\sup _{|w|=t_{k+1}} \frac{\log |f(w)|}{\left|\log \left(1-t_{k+1}\right)\right|} \cdot \frac{\mid \log \left(1-t_{k+1} \mid\right.}{\left|\log \left(1-t_{k}\right)\right|} \\
& \leq(1+\varepsilon) \sup _{|w|=t_{k+1}} \frac{\log |f(w)|}{\left|\log \left(1-t_{k+1}\right)\right|} \leq(1+\varepsilon)\left[T_{f, S}+\varepsilon\right] .
\end{aligned}
$$


Since $\varepsilon>0$ is arbitrary, from the last inequality it follows that $T_{f} \leq T_{f, S}$ for every $f \in A^{-\infty}(\mathbb{B})$, which means that $\mathcal{S}$ is sampling for $A^{-\infty}(\mathbb{B})$.

Question 4.2. Is the condition (18) necessary for $\mathcal{S}$ to be sampling for $A^{-\infty}$ ?

Furthermore, we can prove, in the same manner as in [6], that under a weaker condition for $\left(t_{k}\right)$ the corresponding concentric set is weakly sufficient.

Proposition 4.3. Suppose that $0<\left(t_{k}\right) \uparrow 1$ satisfying the following condition

$$
\limsup _{k \rightarrow \infty} \frac{\left|\log \left(1-t_{k+1}\right)\right|}{\left|\log \left(1-t_{k}\right)\right|}<\infty .
$$

The concentric set

$$
\widetilde{\mathcal{S}}=\bigcup_{k=1}^{\infty}\left\{z \in \mathbb{B}:|z|=t_{k}\right\}
$$

is weakly sufficient for the space $A^{-\infty}(\mathbb{B})$.

Proof. Let

$$
\limsup _{k \rightarrow \infty} \frac{\left|\log \left(1-t_{k+1}\right)\right|}{\left|\log \left(1-t_{k}\right)\right|}=M .
$$

For $M_{1}>M$ there is $k_{0}$ such that for any $k>k_{0}$

$$
1-t_{k+1}>\left(1-t_{k}\right)^{M_{1}} \text {. }
$$

Let $q$ be any natural number which is greater than the integral part of $M_{1} p$, for any $f \in A^{-\infty}$ and $z$ such that $|z|<t_{k_{0}}$ we have

$$
(1-|z|)^{q}|f(z)| \leq \sup _{|z| \leq t_{k_{0}}}|f(z)| \text {. }
$$

So to prove $f \in A^{-q}$ is suffices to show that $(1-|z|)^{q}|f(z)|$ is bounded for $|z| \geq t_{k_{0}}$. Indeed, let $k$ be the unique integer such that $t_{k}<|z| \leq t_{k+1}$. Then we have

$$
(1-|z|)^{q}|f(z)| \leq\left(1-t_{k}\right)^{q} \sup _{|z|=t_{k+1}}|f(z)| \leq\left(1-t_{k+1}\right)^{p} \sup _{|z|=t_{k+1}}|f(z)| \leq\|f\|_{p, \widetilde{\mathcal{S}}} .
$$

This completes the proof.

Question 4.4. Is the condition (19) necessary for $\mathcal{S}$ to be weakly sufficient for $A^{-\infty}$ ?

Remark 4.5. We see that although the two concentric sets $\mathcal{S}$ and $\widetilde{\mathcal{S}}$ have the same structure, they play, by different conditions imposed on the sequence $\left(t_{k}\right)$, different roles for $A^{-\infty}$. In particular, the concentric sampling set $\mathcal{S}$ is also weakly sufficient for the space $A^{-\infty}$.

This type of examples of weakly sufficient sets is in fact a particular case of a general result about the implication "sampling" $\Rightarrow$ "weak sufficiency" which will be treated in the next subsection. 


\subsection{Sampling property implies weak sufficiency}

We now study a relationship between sampling and weakly sufficient sets. First we prove that a weak sufficiency is necessary for a set to be sampling.

Proposition 4.6. Each sampling set for $A^{-\infty}(\mathbb{B})$ is weakly sufficient for this space.

Proof. Let $S \subset \mathbb{B}$ be a sampling set for the space $A^{-\infty}$. We prove that the two conditions in Theorem 3.6 are satisfied.

- The first condition.

Suppose that $f \in A^{-\infty}$ and $f(z)=0 \forall z \in S$. Then

$$
T_{S}(f)=\limsup _{z \in S,|z| \rightarrow 1} \frac{\log |f(z)|}{|\log (1-|z|)|}=-\infty .
$$

As $T(f)=T_{S}(f)$ it follows that

$$
T(f)=\limsup _{z \in \mathbb{B},|z| \rightarrow 1} \frac{\log |f(z)|}{|\log (1-|z|)|}=-\infty .
$$

Then in particular $T(f)$ is negative, which is all we need. There exists a positive number $a$ such that $T(f)<-a$. Hence there exists a positive $\delta$ such that $\log |f(z)| \leq a \log (1-|z|)$ for all $z$ such that $|z| \geq 1-\delta$.

Fix $w \in B$. Then for all $r \geq|w|$ we have $|f(w)| \leq \sup _{|z|=r}|f(z)| \leq(1-r)^{a}$ when $r \geq 1-\delta$. Now let $r$ tend to 1 . We see that we must have $|f(w)|=0$.

Thus if $S$ is a sampling set for $A^{-\infty}$, then it is a set of uniqueness for $A^{-\infty}$.

- The second condition.

Now we prove that $\forall p \exists m=m(p): A^{-p, S} \subset A^{-m}$.

Indeed, take and fix a natural number $p \geq 1$. For an arbitrary element $f \in A^{-p, S}$ we have

$$
\sup _{z \in S}(1-|z|)^{p}|f(z)|=\|f\|_{p, S}<\infty .
$$

From this it follows that

$$
T_{S}(f)=\limsup _{z \in S,|z| \rightarrow 1} \frac{\log |f(z)|}{|\log (1-|z|)|} \leq p,
$$

and therefore,

$$
T(f)=\limsup _{z \in \mathbb{B},|z| \rightarrow 1} \frac{\log |f(z)|}{|\log (1-|z|)|} \leq p .
$$

Hence,

$$
\sup _{z \in \mathbb{B},|z| \rightarrow 1}(1-|z|)^{p+1}|f(z)|<\infty,
$$

that is, $f \in A^{-(p+1)}$, which means that $A^{-p} \subset A^{-(p+1)}$.

Thus if $S$ is a sampling set for $A^{-\infty}$, then it is a set of uniqueness for $A^{-\infty}$, and $\forall p \geq 1: A^{-p} \subset A^{-(p+1)}$. By Theorem 3.6, $S$ is a weakly sufficient set for the space $A^{-\infty}$. The proposition is proved. 


\subsection{Discrete sampling sets}

We already showed that the concentric sets of the types $\mathcal{S}$ and $\widetilde{\mathcal{S}}$ are sampling and weakly sufficient, respectively. Both examples are non-discrete sets. A question arises: how about the existence of discrete sets?

As mentioned in Section 2, in [3] we gave an explicit way to construct a countable weakly sufficient set for $A^{-\infty}$, for every $n \geq 1$. We are now interested in a question of existence of a discrete set which is sampling in this space.

It turns out that the algorithm mentioned above, under some modifications, also provides an explicit construction of sampling sets. In this subsection we present a method to "extract" from the concentric set a discrete sampling subset. These results are obtained in spirit of works $[4,6]$ on similar sets in the one-dimensional case.

Note that in the proof of Proposition 4.1 we did not use the fact that $f \in$ $A^{-\infty}(\mathbb{B})$. In this section we see that the polynomial growth property plays an important role in passing to a discrete case.

We first outline some basic facts from [3] on construction of discrete weakly sufficient sets in $A^{-\infty}$.

For $0<t<1$ denote

$$
\mathcal{S}_{t}=\{z \in \mathbb{B}:|z|=t\} \text { and } \mathcal{M}_{t}(f)=\sup _{z \in \mathcal{S}_{t}}|f(z)| .
$$

Proposition 4.7. For any numbers $0<a<b<1$ and any points $z, w \in \mathcal{S}_{a}$, if $f \in \mathcal{O}(\mathbb{B})$, then

$$
|f(z)-f(w)| \leq \frac{2 n \sqrt{n}|z-w|}{b-a} \mathcal{M}_{b}(f) .
$$

Consider a sequence $0<\left(t_{k}\right) \uparrow 1$ satisfying the following, rather natural in comparison with (18), condition.

$$
\lim _{k \rightarrow \infty} \frac{1-t_{k+1}}{1-t_{k}}=1
$$

Such sequences are abundant. For example, $t_{k}=1-\frac{1}{k+1}, t_{k}=1-e^{-k}, \ldots(k=$ $1,2, \ldots)$ work.

We notice that the condition (20) implies the condition (18). Indeed,

$$
\begin{aligned}
\lim _{k \rightarrow \infty}\left[\frac{\left|\log \left(1-t_{k+1}\right)\right|}{\left|\log \left(1-t_{k}\right)\right|}-1\right] & =\lim _{k \rightarrow \infty}\left[\frac{\log \left(1-t_{k+1}\right)}{\log \left(1-t_{k}\right)}-1\right] \\
& =\lim _{k \rightarrow \infty}\left[\frac{\log \left(1-t_{k+1}\right)-\log \left(1-t_{k}\right)}{\log \left(1-t_{k}\right)}\right] \\
& =\lim _{k \rightarrow \infty}\left[\frac{\log \frac{1-t_{k+1}}{1-t_{k}}}{\log \left(1-t_{k}\right)}\right] \\
& =0(\text { due to }(20)) .
\end{aligned}
$$


In order to avoid complications, we shall use the notation $\mathcal{M}_{k}(f)$ and $\mathcal{S}_{k}$ in place of $\mathcal{M}_{t_{k}}(f)$ and $\mathcal{S}_{t_{k}}$, respectively.

Take a sequence $0<\left(t_{k}\right) \uparrow 1$ with the aforementioned property. Furthermore, take a sequence of natural numbers $\left(s_{k}\right) \uparrow \infty$ so that

$$
\lim _{k \rightarrow \infty} \frac{1}{s_{k}\left(t_{k+1}-t_{k}\right)}=0 \text {. }
$$

Such sequences $\left(s_{k}\right)$ can easily be found.

Fix a natural number $N_{0} \geq 1$. Then, on $\mathcal{S}_{k}$ with $k=N_{0}, N_{0}+1, \ldots$, mark $m_{k}$ points $z_{k, j}\left(j=1,2, \ldots, m_{k}\right)$, which form a $1 / s_{k}$-net on $\mathcal{S}_{k}$.

We renumber these points $\left\{z_{k, j} ; 1 \leq j \leq m_{k}, k \geq N_{0}\right\}$ under one sequence, denoted by $\Lambda=\left(\lambda_{k}\right)_{k=1}^{\infty}$, writing first all points with $k=N_{0}$, and then with $k=N_{0}+1$, etc. Note that the meaning of a choice of the number $N_{0}$ is only in that the elements of $\Lambda$ can be chosen in the ball $\mathbb{B}$ to be "arbitrarily far away" from the origin.

Theorem 4.8. The sequence $\Lambda=\left(\lambda_{k}\right)_{k=1}^{\infty} \subset \mathbb{B}$ constructed above is weakly sufficient for the space $A^{-\infty}(\mathbb{B})$.

As a countable set $\Lambda=\left(\lambda_{k}\right)$ is a subset of the set $\widetilde{\mathcal{S}}$ in Proposition 4.3, Theorem 4.8 shows how to extract from the weakly sufficient concentric set of the type $\widetilde{\mathcal{S}}$ a countable subset that is weakly sufficient for the space $A^{-\infty}$.

This fact gives us an idea how to get a countable sampling subset from the concentric sampling set of the type $\mathcal{S}$ in Proposition 4.1.

For this we choose a sequence of natural numbers $\left(s_{k}\right) \uparrow \infty$ so that the following condition is satisfied.

$$
\lim _{k \rightarrow \infty} \frac{1}{s_{k}\left(t_{k+1}-t_{k}\right)\left(1-t_{k+1}\right)^{p}}=0 \text { for any } p>0 .
$$

Note that this condition is quite stronger than the condition (21).

Such a sequence $\left(s_{k}\right)$, as was for the condition (21), can easily be found. For example, starting from any sequence $\left(\rho_{k}\right)$ of positive real numbers tending to infinity, one can always construct

$$
s_{k}=\text { the intergal part of }\left(\frac{1}{s_{k}\left(t_{k+1}-t_{k}\right)\left(1-t_{k+1}\right)^{\rho_{k}}}\right), k=1,2, \ldots
$$

This $\left(s_{k}\right)$ works for the condition (22), and in its turn, the condition (21) holds.

Then we can construct a weakly sufficient sequence $\Lambda=\left(\lambda_{k}\right)$, that lies on the concentric union $\mathcal{S}=\bigcup \mathcal{S}_{k}$, for the space $A^{-\infty}(\mathbb{B})$.

We are going now to prove that this countable set is sampling for $A^{-\infty}$.

Notice that for each positive number $p$ the quantity $\|f\|_{p}=\sup _{z \in \mathbb{B}}(1-|z|)^{p}|f(z)|$ can be rewritten as follows

$$
\|f\|_{p}=\sup _{0<t<1} \mathcal{M}_{t}(f)(1-t)^{p} .
$$


From this it follows that

$$
\forall 0<t<1, \mathcal{M}_{t}(f) \leq \frac{\|f\|_{p}}{(1-t)^{p}} .
$$

Assume that there exists $f_{0} \in A^{-\infty}(\mathbb{B})$ such that $T_{f_{0}, \Lambda}<T_{f_{0}}$. Take $a, b$ such that

$$
T_{f_{0}, \Lambda}<a<b<T_{f_{0}}
$$

This implies that

$$
\exists \rho \in(0,1): \frac{\log \left|f_{0}(z)\right|}{|\log (1-|z|)|}<a, \forall z \in \Lambda,|z|>\rho,
$$

and

$$
\forall \rho \in(0,1), \exists z^{\prime} \in \mathbb{B},\left|z^{\prime}\right|>\rho: \frac{\log \left|f_{0}\left(z^{\prime}\right)\right|}{\left|\log \left(1-\left|z^{\prime}\right|\right)\right|}>b .
$$

Thus the inequalities above can be rewritten as follows.

$$
\exists \rho \in(0,1):\left|f_{0}(z)\right|<\frac{1}{(1-|z|)^{a}}, \forall z \in \Lambda,|z|>\rho,
$$

and

$$
\forall \rho \in(0,1) \exists z^{\prime} \in \mathbb{B},\left|z^{\prime}\right|>\rho:\left|f_{0}\left(z^{\prime}\right)\right|>\frac{1}{\left(1-\left|z^{\prime}\right|\right)^{b}} .
$$

Our main procedure starts from these important points, conditions (24) and $(25)$.

- Step 1: By the condition (24)

$$
\exists \rho_{1} \in(0,1):\left|f_{0}(z)\right|<\frac{1}{(1-|z|)^{a}}, \forall z \in \Lambda,|z|>\rho_{1} .
$$

For such a $\rho_{1}$, by the condition $(25)$

$$
\exists z_{1} \in \mathbb{B},\left|z_{1}\right|>\rho_{1}:\left|f_{0}\left(z_{1}\right)\right|>\frac{1}{\left(1-\left|z_{1}\right|\right)^{b}} .
$$

Take the unique natural number $k$ so that $t_{k-1} \leq\left|z_{1}\right|<t_{k}$.

Since $f_{0} \in A^{-\infty}$ there exists a positive integer $p_{0}$ such that $f_{0} \in A^{-p_{0}}(\mathbb{B})$. In this case

$$
\mathcal{M}_{k+1}\left(f_{0}\right) \leq \frac{\left\|f_{0}\right\|_{p_{0}}}{\left(1-t_{k+1}\right)^{p_{0}}}
$$

by $(23)$.

Let $w_{k} \in \mathcal{S}_{k}$ with $\left|f_{0}\left(w_{k}\right)\right|=\mathcal{M}_{k}\left(f_{0}\right)$. Then by the construction of points $z_{k, j}\left(j=1, \ldots, m_{k}\right)$, there exists $z_{k, j_{0}} \in \mathcal{S}_{k}$ such that $\left|w_{k}-z_{k, j_{0}}\right|<\frac{1}{s_{k}}$. 
It then follows by Proposition 4.7 that

$$
\begin{aligned}
\mathcal{M}_{k}\left(f_{0}\right)-\left|f_{0}\left(z_{k, j_{0}}\right)\right| & =\left|f_{0}\left(w_{k}\right)\right|-\left|f_{0}\left(z_{k, j_{0}}\right)\right| \\
& \leq\left|f_{0}\left(w_{k}\right)-f_{0}\left(z_{k, j_{0}}\right)\right| \\
& \leq \frac{2 n \sqrt{n}\left|w_{k}-z_{k, j_{0}}\right|}{t_{k+1}-t_{k}} \mathcal{M}_{k+1}\left(f_{0}\right) \\
& \leq \frac{2 n \sqrt{n} \mathcal{M}_{k+1}\left(f_{0}\right)}{s_{k}\left(t_{k+1}-t_{k}\right)}
\end{aligned}
$$

Hence,

$$
\mathcal{M}_{k}\left(f_{0}\right) \leq\left|f_{0}\left(z_{k, j_{0}}\right)\right|+\frac{2 n \sqrt{n}}{s_{k}\left(t_{k+1}-t_{k}\right)} \mathcal{M}_{k+1}\left(f_{0}\right) .
$$

Notice that $z_{k, j_{0}} \in \mathcal{S}_{k} \bigcap \Lambda$, thus one induces from (26) that

$$
\left|f_{0}\left(z_{k, j_{0}}\right)\right|<\frac{1}{\left(1-\left|z_{k, j_{0}}\right|\right)^{a}}=\frac{1}{\left(1-t_{k}\right)^{a}} .
$$

Therefore,

$$
\begin{aligned}
\left|f_{0}\left(w_{k}\right)\right| & \leq \frac{1}{\left(1-t_{k}\right)^{a}}+\frac{2 n \sqrt{n}}{s_{k}\left(t_{k+1}-t_{k}\right)} \mathcal{M}_{k+1}\left(f_{0}\right) \\
& \leq \frac{1}{\left(1-t_{k}\right)^{a}}+\frac{2 n \sqrt{n}\left\|f_{0}\right\|_{p_{0}}}{s_{k}\left(t_{k+1}-t_{k}\right)\left(1-t_{k+1}\right)^{p_{0}}} .
\end{aligned}
$$

As $t_{k-1} \leq\left|z_{1}\right|<t_{k}$, we can choose $z_{2} \in S_{k}$ so that $\left|f_{0}\left(z_{1}\right)\right| \leq\left|f_{0}\left(z_{2}\right)\right|$. Then we arrive at

$$
\left|f_{0}\left(z_{2}\right)\right| \geq\left|f_{0}\left(z_{1}\right)\right|>\frac{1}{\left(1-\left|z_{1}\right|\right)^{b}} \geq \frac{1}{\left(1-t_{k-1}\right)^{b}}
$$

and

$$
\left|f_{0}\left(z_{2}\right)\right|<\left|f_{0}\left(w_{k}\right)\right| \leq \frac{1}{\left(1-t_{k}\right)^{a}}+\frac{2 n \sqrt{n}\left\|f_{0}\right\|_{p_{0}}}{s_{k}\left(t_{k+1}-t_{k}\right)\left(1-t_{k+1}\right)^{p_{0}}} .
$$

Thus we obtain the following inequalities

$$
\frac{1}{\left(1-t_{k-1}\right)^{b}}<\left|f_{0}\left(z_{1}\right)\right| \leq\left|f_{0}\left(z_{2}\right)\right| \leq \frac{1}{\left(1-t_{k}\right)^{a}}+\frac{\left.2 n \sqrt{n}|| f_{0}\right|_{p_{0}}}{s_{k}\left(t_{k+1}-t_{k}\right)\left(1-t_{k+1}\right)^{p_{0}}} .
$$

By the condition (22), the sequence $\left(\frac{1}{s_{k}\left(t_{k+1}-t_{k}\right)\left(1-t_{k+1}\right)^{p_{0}}}\right)$ is bounded. Therefore, from the last inequalities we get

$$
\frac{1}{\left(1-t_{k-1}\right)^{b}} \leq \frac{1}{\left(1-t_{k}\right)^{a}}+C_{0}
$$

for some positive constant $C_{0}$ depending on $p_{0}$. Hence,

$$
\left(\frac{1}{1-t_{k-1}}\right)^{b-a} \leq\left(\frac{1-t_{k-1}}{1-t_{k}}\right)^{a}+C_{0}\left(1-t_{k-1}\right)^{a}
$$


Thus we can find a natural number $k$, denoted in this Step 1 by $k_{1}$ such that

$$
\left(\frac{1}{1-t_{k_{1}-1}}\right)^{b-a} \leq\left(\frac{1-t_{k_{1}-1}}{1-t_{k_{1}}}\right)^{a}+C_{0}\left(1-t_{k_{1}-1}\right)^{a}
$$

- Step 2: Consider the condition (25) for $\rho_{2} \geq\left|z_{2}\right|$ in Step 1; do the same as in Step 1, we see that there exists a corresponding $z_{1}^{\prime}$ with $\left|z_{1}^{\prime}\right|>\rho_{2}$ such that $\left|f_{0}\left(z_{1}^{\prime}\right)\right|>\frac{1}{\left(1-\left|z_{1}^{\prime}\right|\right)^{b}}$.

Again take $k$ so that $t_{k-1} \leq\left|z_{1}^{\prime}\right|<t_{k}$ and we have $z_{2}^{\prime},\left|z_{2}^{\prime}\right|>\rho_{2}$ with

and

$$
\left|f_{0}\left(z_{2}^{\prime}\right)\right| \geq\left|f_{0}\left(z_{1}^{\prime}\right)\right|>\frac{1}{\left(1-\left|z_{1}^{\prime}\right|\right)^{b}} \geq \frac{1}{\left(t_{k-1}\right)^{b}}
$$

$$
\left|f_{0}\left(z_{2}^{\prime}\right)\right|<\frac{1}{\left(1-\left|z_{2}^{\prime}\right|\right)^{a}} \leq \frac{1}{\left(1-t_{k}\right)^{a}}+C_{0} .
$$

At this Step 2, denote such $k$ by $k_{2}$, we notice that $k_{2}$ can be chosen so that $k_{1}<k_{2}$, and we arrive at

$$
\left(\frac{1}{1-t_{k_{2}-1}}\right)^{b-a} \leq\left(\frac{1-t_{k_{2}-1}}{1-t_{k_{2}}}\right)^{a}+C_{0}\left(1-t_{k_{2}-1}\right)^{a} .
$$

Now it is very important for us to notice that one can always repeat this process, and end up with a monotonically increasing sequence $\left(k_{\ell}\right)$ of positive integers diverging to infinity with

$$
\left(\frac{1}{1-t_{k_{\ell}-1}}\right)^{b-a} \leq\left(\frac{1-t_{k_{\ell}-1}}{1-t_{k_{\ell}}}\right)^{a}+C_{0}\left(1-t_{k_{\ell}-1}\right)^{a} .
$$

Letting $\ell \rightarrow \infty$ in (28), we then see that the left-hand-side diverges to infinity ( since $b>a$ ), but the right-hand-side converges to 1 : a contradiction.

Thus we obtain the following result.

Theorem 4.9. The sequence $\Lambda=\left(\lambda_{k}\right)$ constructed above, with $\left(s_{k}\right)$ satisfying the condition $(22)$, is sampling for the space $A^{-\infty}(\mathbb{B})$.

Acknowledgment. The author thanks Kang-Tae Kim for fruitful discussions, Christer Kiselman and Emil Straube for references as well as useful correspondence. He also thanks the referees for their remarks and comments that lead to the improvement of this paper.

\section{References}

[1] A. V. Abanin, Some criteria for weak sufficiency, Mat. Zametki 40 (1986), no. 4, 442454; English translation in: Math. Notes 40 (1986), no. 3-4, 757-764.

[2] J. Bonet and P. Domański, Sampling sets and sufficient sets for $A^{-\infty}$, J. Math. Anal. Appl. 277 (2003), no. 2, 651-669.

[3] Y. J. Choi, L. H. Khoi, and K. T. Kim, On an explicit construction of weakly sufficient sets for the function algebra $A^{-\infty}(\Omega)$, Complex Var. Elliptic Equ. 54 (2009), no. 9, 879-897. 
[4] C. A. Horowitz, B. Korenblum, and B. Pinchuk, Sampling sequences for $A^{-\infty}$, Michigan Math. J. 44 (1997), no. 2, 389-398.

[5] V. Ganapathy Iyer, On effective sets of points in relation to integral functions, Trans. Amer. Math. Soc. 42 (1937), no. 3, 358-365; A correction to the paper: Trans. Amer. Math. Soc. 43 (1938), no. 3, 494.

[6] L. H. Khoi and P. J. Thomas, Weakly sufficient sets for $A^{-\infty}(\mathbf{D})$, Publ. Mat. 42 (1998), no. $2,435-448$.

[7] H. Komatsu, Projective and injective limits of weakly compact sequences of locally convex spaces, J. Math. Soc. Japan 19 (1967), 366-383.

[8] X. Massaneda, Interpolation by holomorphic functions in the unit ball with polynomial growth, Ann. Fac. Sci. Toulouse Math. (6) 6 (1997), no. 2, 277-296.

[9] $\_A^{-\infty}$-interpolation in the ball, Proc. Edinburgh Math. Soc. (2) 41 (1998), no. $2,359-367$.

[10] E. J. Straube, Harmonic and analytic functions admitting a distribution boundary value, Ann. Scuola Norm. Sup. Pisa Cl. Sci. (4) 11 (1984), no. 4, 559-591.

Division of Mathematical Sciences

School of Physical and Mathematical Sciences

NANyAng TeChNological University (NTU)

637371 SingAPORE

E-mail address: 1hkhoi@ntu.edu.sg 